\title{
Actualización del catálogo de Culicoides Latreille, 1809 (Diptera, Ceratopogonidae) de España
}

\author{
P. M. Alarcón-Elbal* \& J. Lucientes*
}

Las enfermedades asociadas a artrópodos continúan constituyendo en la actualidad un gran problema en muchos países, especialmente en los subdesarrollados, afectando a la población humana y animal. Nuevas estirpes de patógenos, así como nuevos agentes causantes de enfermedades transmitidos por artrópodos, están originando serios problemas de salud local e internacional.

Un claro ejemplo de ello es el virus Schmallenberg, detectado recientemente en Europa. Este orbivirus parece afectar mayoritariamente al ganado ovino, bovino y caprino, produciendo malformaciones congénitas y provocando fiebre, diarrea severa y abortos, entre algunas de las manifestaciones más importantes descritas hasta el momento. Desde su identificación por vez primera en Alemania en noviembre de 2011, en la Unión Europea (UE) se han encontrado nuevos casos, siendo el país de origen el que más brotes ha constatado y seguido por Francia, Bélgica, Holanda, Reino Unido, Luxemburgo, Italia y España (MAGRAMA, 2012). Como otras arbovirosis que afectan al ganado tales como la Lengua Azul, este virus parece ser transmitido por dípteros nematóceros del género Culicoides Latreille, 1809 (Diptera, Ceratopogonidae), siendo muchos los estudios que se están realizando en la actualidad para conocer mejor su mecanismo de propagación y todos los factores implicados.

Estos pequeños artrópodos no solo pueden actuar como vectores de arbovirus, sino también de nematodos y protozoos, todos ellos causantes de enfermedades en los animales, pero también excepcionalmente en las personas. Además, los daños directos producto de su picadura originan fuertes reacciones alérgicas y producen pérdidas económicas importantes en sectores relacionados con actividades al aire libre (Blackwell et al., 1997; Takken \& Knols, 2010).

En Europa tienen especial relevancia aquellas patogenias vehiculizadas por estos insectos que afectan al ganado. Además de la ya mencionada Lengua Azul, otras como la Peste Equina Africana de los caballos ya se ha registrado dentro de nuestras fronteras en el pasado (OIE, 2010). Aun así, no puede descartarse que otras enfermedades de distribución tropical o subtropical puedan acabar circulando por Europa, especialmente debido al calentamiento global, ya que muchos vectores están experimentando una expansión geográfica notable (Meiswinkel et al., 2008).

La distribución geográfica de los vectores de enfermedades es un aspecto de reconocida importancia para la evaluación epidemiológica en los programas de control, siendo ampliamente conocida la influencia de factores ambientales sobre la distribución y abundancia de insectos (Gorla, 2002). Es por ello que este trabajo acomete una actualización del catálogo de Culicoides descrito en España hasta mayo de 2012, haciendo especial énfasis en la cronología de los descubrimientos que sobre este género de dípteros se ha anotado en el transcurso de más de un siglo de investigación en el país.

Los primeros registros de individuos del género Culicoides en España se deben al presbítero católico de origen austriaco Gabriel Strobl (1846-1925), el cual no solo realizó importantes aportaciones en el campo de la entomología sino también en el de la

* Departamento de Patología Animal, Unidad de Parasitología y Enfermedades Parasitarias. Facultad de Veterinaria. Universidad de Zaragoza. España. C/ Miguel Servet 177 - 50013 Zaragoza - Tel: +34 976761560 - E-mail: pedro.alarcon@uv.es 
botánica. Strobl (1900) realiza estudios de entomofauna en Andalucía (Sierra Nevada) y Cataluña, incorporando las primeras especies para España:

- Culicoides pulicaris (Linnaeus, 1758) (nombrado como Ceratopogon pulicari Linnaeus, 1758)

- Culicoides punticollis Becker, 1903 (nombrado como Ceratopogon algecirensis Strobl, 1900 - Nombre suprimido por ICZN Opinion 1643)

Un lustro después, Strobl (1906) prosigue sus estudios en estas comunidades autónomas y encuentra una nueva especie:

- Culicoides obsoletus Meigen, 1918 (nombrado como Ceratopogon varius Winnertz, 1825)

Antes de acabar la primera década del siglo XX, Czerny \& Strobl (1909) relegan Ceratopogon algecirensis a variedad de Ceratopogon (Culicoides) pulicaris.

No fue hasta 1912 cuando, de la mano de Arias Encobet, se publica el primer catálogo de los dípteros presentes en España. El trabajo incluye una recopilación de lo divulgado hasta el año 1909, éste inclusive. Esta obra de referencia ya hace mención a los individuos encontrados por Strobl (1900, 1906) como: pulicaris L. Ceratopogon - Sierra Nevada (Strobl); Malgrat (Strobl), pulicaris $L$. var. algecirasensis Strobl. Algeciras (Strobl) y varius Winn. Ceratopogon - Algeciras, Malgrat (Strobl). Nótese en este caso la pequeña diferencia nomenclaturial que atribuye este autor a $C$. algecirensis, nombrándola erróneamente $C$. algecirasensis.

Tuvieron que pasar varias décadas hasta que la investigación de este taxón fuera retomada, hecho debido en parte a que los dipterólogos de la época realizaban en su mayoría trabajos encaminados hacia la tan necesaria lucha antipalúdica y los anofelinos. Tras este parón, el cual abarca medio siglo, entra en escena Juan Gil Collado, ilustre entomólogo jienense quien, en 1929 y tras sus años postdoctorales, recibe el título otorgado por la Dirección General de Sanidad de Entomólogo Facultativo de la lucha antipalúdica. En un estudio realizado en Badajoz, De Prada \& Gil Collado (1959) citan 11 especies, 8 de ellas descritas por primera vez en España, y añaden algunas observaciones sobre éstas proponiendo una primera clave para su determinación. Las nuevas especies fueron:

- Culicoides circumscriptus Kieffer, 1918

- Culicoides fascipennis Steager, 1839

- Culicoides impunctatus Goetghebuer, 1920
- Culicoides nubeculosus Meigen, 1818

- Culicoides festivipennis Austen, 1921 (nombrado como Culicoides odibilis Austen, 1921)

- Culicoides parroti Kieffer, 1922

- Culicoides pictipennis (Staeger, 1839)

- Culicoides riethi Kieffer, 1913

En la década de los setenta dos estudios permiten incrementar el número de especies descritas de 11 a 16. En el primero de ellos, Callot et al. (1975) añaden 3 especies, encontradas en un estudio previo en 1970 en la provincia valenciana de Alicante:

- Culicoides catanei Clastrier, 1957

- Culicoides jumineri Callot \& Kremer, 1969

- Culicoides maritimus Kieffer, 1924 (nombrado como Culicoides submaritimus Dzhafarov, 1957)

Por su parte, Sánchez-Covisa et al. (1979) descubren en los alrededores de Madrid las siguientes especies arborícolas:

- Culicoides fagineus Edwards, 1939

- Culicoides haranti Rioux, Descous \& Pech, 1959

El punto de inflexión en el conocimiento de estos dípteros en España está marcado sin duda por la década de los ochenta, momento en el que numerosos estudiosos retoman el campo viendo la luz algunos trabajos que ayudan a comprender la biología y la distribución de estos dípteros en el país. Sahuquillo Herráiz \& Gil Collado (1982a) describen 7 nuevas especies para España en Navarra:

- Culicoides accraensis Carter, Ingram \& Macfie, 1920

- Culicoides kibunensis Tokunaga, 1937 (nombrado como Culicoides cubitalis Edwards, 1939)

- Culicoides newsteadi Austin, 1921 (nombrado como Culicoides halophilus Kieffer, 1924)

- Culicoides minutissimus (Zettersted, 1865)

- Culicoides subfasciipennis Kieffer, 1925

- Culicoides truncorum Edwards, 1939 (nombrado como Culicoides sylvarum Callot \& Kremer, 1961)

- Culicoides vexans (Staeger, 1839)

Fruto del estudio de un macho sin determinar recogido por De Prada \& Gil Collado (1959) en Badajoz, Sahuquillo Herráiz \& Gil Collado (1982b) describen una nueva especie para España y para Europa:

- Culicoides sahariensis Kieffer, 1923

Havelka (1982) cita dos nuevas especies en el noreste de España, en la provincia de Gerona. Este trabajo no ve la luz hasta 1983, por lo que no puede 
discutirse la primera autoría de $C$. minutissimus a Sahuquillo Herráiz \& Gil Collado (1982a), también encontrada por Havelka pero descrita con anterioridad por éstos. Las especies nuevas, y que elevan a 26 el número total, son:

- Culicoides punctatus (Meigen, 1804)

- Culicoides longipennis Khalaf, 1957

Gil Collado \& Sahuquillo Herráiz (1983) hacen una primera revisión de la fauna de Culicoides en diversas regiones de España. En su trabajo señalan 12 nuevas citas para la España peninsular, aunque en la tabla resumen el número es de 13 , hecho que se explica de la suma de las 7 especies encontradas por Sahuquillo Herráiz \& Gil Collado (1982a), la cita de $C$. sahariensis de ese mismo año (Sahuquillo Herráiz \& Gil Collado, 1982b) y las que realmente aporta su nuevo trabajo. Aun así, estos datos se refieren en su gran mayoría a los hallazgos que Sahuquillo Herráiz (1981) refleja en su Tesis Doctoral, fruto del muestreo en las provincias de Valencia y Navarra. Hay que apuntar que Gil Collado \& Sahuquillo Herráiz (1983) no tienen en cuenta el trabajo de Havelka (1982), por lo que también consideran $C$. longipennis como primera cita. Realmente este trabajo aporta 4 nuevas especies, las cuales hacen ascender a 30 el número total:

- Culicoides almeidae Cambournac, 1970

- Culicoides corsicus Kremer, LeBerre \& BeaucournuSaguez, 1971

- Culicoides duddingstoni Kettle \& Lawson, 1955

- Culicoides segnis Campbell \& Clinton, 1959

Ese mismo año, Mellor et al. (1983) amplían el listado en 10 especies, centrando por primera vez el estudio en el rol como vectores de arbovirus de estos dípteros. Es este el primer artículo en el que se informa de la presencia del que sería principal vector de la Lengua Azul en nuestro país, Culicoides imicola, aunque la enfermedad era considerada entonces exótica para Europa. Las especies que se añaden al catálogo son:

- Culicoides brunnicans Edwards, 1939

- Culicoides gejgelensis Dzhafarov, 1964

- Culicoides griseidorsum Kieffer, 1818 (nombrado como Culicoides saevanicus Dzhafarov, 1960)

- Culicoides heteroclitus Kremer \& Callot, 1965

- Culicoides imicola Kieffer, 1913

- Culicoides kurensis Dzhafarov, 1960

- Culicoides odiatus Austen, 1921

- Culicoides saevus Kieffer, 1922
- Culicoides shaklawensis Khalaf, 1957

- Culicoides vidourlensis Callot, Kremer, Molet \& Bach, 1968

El último trabajo reportado en esta década (Gil Collado \& Sahuquillo Herráiz, 1985), aunque no supone ninguna nueva incorporación al listado de especies, proporciona una interesante primera clave de identificación de los ceratopogónidos de España. Aun así, el número se incrementa en más del doble en esta década, alcanzando las 40 especies.

En la década de los noventa, el primer estudio realizado por parte del entonces Ministerio de Agricultura, Pesca y Alimentación sobre la distribución y prevalencia de estos dípteros añade 5 especies nuevas (Anónimo, 1992):

- Culicoides begueti Clastrier, 1957

- Culicoides chiopterus (Meigen, 1830)

- Culicoides helveticus Callot, Kremer \& Deduit, 1962

- Culicoides poperinghensis Goetghebuer, 1953

- Culicoides univittatus Vimmer, 1932

Ese mismo año, en el $\mathrm{V}$ congreso Ibérico de Entomología celebrado en Lisboa, Capela et al. (1992) citan una nueva especie para España y Portugal:

- Culicoides achrayi Kettle \& Lawson, 1955

Un año más tarde, Ortega (1993) suma 2 especies más, encontradas en esta ocasión en Andalucía, aumentando el total a 48:

- Culicoides bahrainensis Boorman, 1989

- Culicoides marcleti Callot, Kremer \& Basset, 1968

Rawlings (1996) publica una clave para la identificación de los Culicoides de la Península Ibérica utilizando un sistema simplificado basado en el patrón de manchas alares, tan característico de estos nematóceros. El objetivo del trabajo era que las claves pudieran servir como el instrumento más importante para desarrollar sistemas informáticos de reconocimiento de géneros, familias y especies de insectos por medio de imágenes. Además, hace una revisión completa de las especies descubiertas hasta la fecha para España y Portugal, añadiendo una nueva cita en Asturias, atribuida a P. Rawlings $\&$ M.A. Abella (unpub), publicación que no alcanza nunca a ver la luz. Esta especie aquí mencionada por primera vez es:

- Culicoides dewulfi Goetghebuer, 1936 
En los dos años posteriores, el entomólogo francés Jean-Claude Delécolle y sus colaboradores comienzan a estudiar los ceratopogónidos de la Península Ibérica, y es gracias a esto que hacen su primera aportación en Delécolle et al. (1997), describiendo la especie número 50 :

- Culicoides brevifrontis Smatov \& Isimbekov, 1971

El holandés Art Borkent cita por primera vez una especie en la España insular, concretamente en Borkent (1997), tras hacer una reexaminación de los ejemplares encontrados por Santos Abreu (1918), apuntando para las Islas Canarias:

- Culicoides analis Santos Abreu, 1918

Delécolle \& Ortega (1998) descubren una nueva especie para el mundo en España, muy similar a $C$. fagineus, aunque con ligeras diferencias morfológicas:

- Culicoides subfagineus Delécolle \& Ortega (1988)

Ortega et al. (1999) en un estudio sobre las especies más abundantes de Andalucía realizado en 1990 y 1991, añaden otra especie, cerrando esta década con un total de 53:

- Culicoides scoticus Downes \& Kettle, 1952

En este trabajo, Ortega et al. (1999) anotan por primera vez cuatro especies cuyas primeras citas ya se encontraban en la literatura, pues nombran a Culicoides agathensis Callot, Kremer \& Rioux 1963, que es sinónimo de C. univitattus y que ya había sido descrito por Anónimo (1992). También se incluyen $C$. bahrainensis y C. marcleti, descritos en Ortega (1993), hecho este que puede explicarse, más siendo la misma persona autor de ambos trabajos, por no considerarse "formal" la cita de 1993 al haberse realizado ésta en una congreso. Finalmente, también aparece como nueva especie C. odiatus, el cual había sido descrito por Mellor et al. (1993).

El nuevo siglo se inicia con la noticia de la declaración de un brote de Lengua Azul en las Islas Baleares durante los meses de septiembre y octubre de 2000. Cuatro años después la enfermedad se reporta en la Península Ibérica, afectando en octubre de 2004 a las comunidades autónomas de Andalucía y Extremadura y a la ciudad de Ceuta, dejando tras más de una década un balance negativo considerable debido a las miles de muertes producidas en la cabaña ganadera y las pérdidas económicas derivadas de la vigilancia y el control de la enfermedad. Es debido a esta circunstancia que desde principios del siglo XXI el estudio de los dípteros del género Culicoides cobra una gran relevancia en nuestro país.

Delécolle (2002) cita 8 especies nuevas en otra nueva obra de referencia para la dipterología española, el Catálogo de Dípteros de España:

- Culicoides montanus Shakirzjanova, 1962

- Culicoides dzhafarovi Remm, 1967

- Culicoides malevillei Kremer \& Coluzzi, 1971

- Culicoides santonicus Callot, Kremer, Rault \& Bach, 1966

- Culicoides simulator Edwards, 1939

- Culicoides submaritimus Dzhafarov, 1962

- Culicoides picturatus Kremer \& Deduit, 1961

- Culicoides pseudoheliophilus Callot \& Kremer, 1961 (nombrada como Culicoides albihalteratus Goetghebuer, 1935)

Las dos últimas especies, aunque no las nombra como primeras citas, son en esta ocasión la primera vez que aparecen en la bibliografía para España. En este trabajo se nombran un total de 53 especies, incluyendo C. submaritimus, ya que reconsidera la sinonimia de ésta con $C$. maritimus, dejando la puerta abierta a que ambas sean subespecies por lo que aparecen separadas. Casi una década después, Talavera et al. (2011) las definen como dos especies diferentes, basándose tanto en diferencias morfológicas como en estudios moleculares.

Delécolle (2002) no nombra a C. accraensis (descrito en Sahuquillo Herráiz \& Gil Collado, 1982a), C. griseidorsum (descrito en Mellor et al., 1983), C. helveticus y C. poperinghensis (descritos en Anónimo, 1992), C. achrayi (descrito por Capela et al., 1992) у C. bahrainensis y $C$. marcle$t i$ (descritos en Ortega, 1993). Prueba de ello es que cita a $C$. begueti como primera nota en España, cuando había sido encontrada una década antes por Anónimo (1992). Tampoco cita a C. dewulfi (apuntado en Rawlings, 1996). Partiendo de este punto y bajo estas consideraciones, son 61 las especies descritas en este momento tras el análisis de Delécolle (2002).

Sarto i Monteys \& Saiz-Ardanaz (2003) descubren 2 nuevas especies, siendo la primera nueva cita para España y la segunda para la Península Ibérica:

- Culicoides pallidicornis Kieffer, 1919

- Culicoides flavipulicaris Dzhafarov, 1964 
Fruto del trabajo de vigilancia entomológica contra la Lengua Azul en Cataluña, Ventura et al. (2005) encuentran 2 nuevas citas para la Península Ibérica. En este trabajo se apunta por vez primera el hecho de una discriminación específica por técnicas de diagnóstico molecular. Las especies nuevas fueron:

- Culicoides deltus Edwards, 1939

- Culicoides alazanicus Dzhafarov, 1961

Goldarazena et al. (2008) reportan por primera vez $C$. imicola en el País Vasco, pero gracias a su estudio en relación a la Lengua Azul en el norte de España, descubren 3 especies desconocidas hasta el momento para la Península:

- Culicoides lupicaris Downes \& Kattle, 1952

- Culicoides comosioculatus Tokunaga, 1956

- Culicoides stigma (Meigen, 1818)

En Cataluña, Sarto i Monteys et al. (2009) descubren 2 especies que, aunque citadas anteriormente en Portugal, no se habían reportado en España, incrementando el número total a 70 :

- Culicoides derisor Callot \& Kremer, 1965

- Culicoides furcillatus Callot, Kremer \& Paradis, 1962

Por otra parte, el trabajo desarrollado por González \& Goldarazena (2011) en diversas localidades del País Vasco permite elevar a 75 este número. Este trabajo está recogido en un libro que recopila gran cantidad de material visual y aporta una guía práctica para su identificación y control, el primer trabajo con este formato sobre Culicoides en nuestro país. Las especies nuevas son:

- Culicoides semimaculatus Clastrier, 1958

- Culicoides heliophilus Edwards, 1921

- Culicoides clastrieri Callot, Kremer \& Deduit, 1962

- Culicoides paradisionensis Boorman, 1988

- Culicoides tauricus Gutsevich, 1959

También nombran a $C$. picturatus y $C$. comosioculatus como primeras citas, pero Delécolle (2002) en el Catálogo de Dípteros de España ya había apuntado la primera y Goldarazena et al. (2008), la segunda.

En ese mismo año, en Cataluña, Talavera et al. (2011) encuentran 5 nuevas especies, siendo la primera especie el primer registro para Europa, la segunda y la tercera para la Península Ibérica, y la cuarta y quinta para España:
- Culicoides yemenensis (Boorman, 1989)

- Culicoides coluzzii Callot, Kremer \& Bailly-Choumara, 1970

- Culicoides sejfadinei Dzhafarov, 1958

- Culicoides pseudopallidus Khalaf, 1961

- Culicoides indistinctus Khalaf, 1961

Estos autores proponen realizar una revisión de la sinonimia de $C$. odiatus y $C$. indistinctus Khalaf, 1961, propuesto por Remm (1981), por lo que ambas especies aparecen separadas por primera vez. También ponen en duda la sinonimia de $C$. sahariensis y C. coluzzi, propuesta por Boorman et al. (1989) y Rawlings (1996), ya que sus estudios morfológicos y moleculares detectan diferencias entre estos individuos, por lo que no están considerados aquí como una misma especie.

Estrada et al. (2011) obtienen a finales de ese mismo año la primera cita en la Península Ibérica de una especie de distribución reciente entre los países mediterráneos. Diversos autores han discutido si realmente se trata de Culicoides jamaicensis Edwards, 1922, habiéndose podido introducir en Europa 500 años atrás gracias a los viajes de Cristóbal Colón (Meiswinkel et al., 2004), siendo esta nueva especie:

- Culicoides paolae Boorman, 1996

Romón et al. (2012) proponen 3 especies nuevas para la Península Ibérica a esta lista, pero todas ellas ya habían sido nombradas anteriormente y no pueden incorporarse como tal a la actualización. La primera de ellas, $C$. riethi, había sido nombrado por De Prada \& Gil Collado (1959) en Badajoz y recogido en anteriores actualizaciones y trabajos, tales como Gil Collado \& Sahuquillo Herráiz (1983) y González \& Goldarazena (2011), trabajo éste último que también recoge la primera cita de $C$. semimaculatus en el País Vasco, también considerada por Romón et al. (2012) erróneamente como nueva cita. Por último, también listan en esta categoría a $C$. indistinctus, pero los primeros en recogerla como una especie distinta a $C$. odiatus son Talavera et al. (2011), un año antes en Cataluña.

Con toda seguridad, estas imprecisiones a la hora de otorgarse nuevas citas en trabajos tan próximos en el tiempo, como es este caso o el de Sahuquillo Herráiz \& Gil Collado (1982a) y Havelka (1982), son inherentes al proceso de evaluación y revisión de los trabajos científicos, el cual es sabido puede durar varios meses (en el mejor de 
Tabla 1.- Listado de especies de Culicoides presentes en España, agrupado por subgéneros y por orden alfabético.

Table 1.- List of Culicoides species present in Spain, grouped by subgenera and in alphabetical order.

\section{ESPECIES ORDENADAS POR SUBGÉNEROS}

AUTORES DE LAS PRIMERAS CITAS PARA ESPAÑA

\section{Avaritia Fox, 1955}

C. chiopterus (Meigen, 1830)

C. dewulfi Goethebuer, 1936

C. imicola Kieffer, 1913

C. montanus Shakirzjanova, 1962

C. obsoletus (Meigen, 1818)

C. scoticus Downes \& Kettle, 1952

Anónimo (1992)

Rawlings (1996)

Mellor et al. (1983)

Delécolle (2002)

Strobl (1906)

Ortega et al. (1999)

\section{Beltranmyia Vargas, 1953}

C. brevifrontis Smatov \& Isimbekov, 1971

C. circumscriptus Kieffer, 1918

Delécolle et al. (1997) De Prada \& Gil Collado (1959)

\section{Culicoides Latreille, 1809}

C. almeidae Cambournac, 1970

C. deltus Edwards, 1939

C. fagineus Edwards,1939

C. flavipulicaris Dzhafarov, 1964

C. impunctatus Goetghebuer, 1920

C. Iupicaris Downes \& Kattle, 1952

C. newsteadi Austen, 1921

C. pulicaris (Linnaeus, 1758)

C. punctatus (Meigen, 1818)

C. subfagineus Delécolle \& Ortega, 1999

Monoculicoides Khalaf, 1954

C. helveticus Callot, Kremer \& Deduit, 1962

C. nubeculosus (Meigen, 1830)

C. parroti Kieffer, 1922

C. puncticollis (Becker, 1903)

C. riethi Kieffer, 1914

C. stigma (Meigen, 1818)

\section{Oecacta Poey, 1853}

C. alazanicus Dzhafarov, 1961

C. begueti Clastrier, 1957

C. brunnicans Edwards, 1939

C. cataneii Clastrier, 1957

C. clastrieri Callot, Kremer \& Deduit, 1962

C. comosioculatus Tokunaga, 1956

C. derisor Callot \& Kremer, 1965

C. duddingstoni Kettle \& Lawson, 1955

C. dzhafarovi Remm, 1967

C. festivipennis Kieffer, 1914

C. furcillatus Callot, Kremer \& Paradis, 1962

C. gejgelensis Dzhafarov, 1964

C. griseidorsum Kieffer, 1818

C. haranti Rioux, Descous \& Pesch, 1959

C. heliophilus Edwards, 1921

C. heteroclitus Kremer \& Callot, 1965

C. jumineri Callot \& Kremer, 1969

C. kibunensis Tokunaga, 1937

C. kurensis Dzhafarov, 1960

C. longipennis Khalaf, 1957

C. malevillei Kremer \& Coluzzi, 1971

C. maritimus Kieffer, 1924
Gil Collado \& Sahuquillo (1983)

Ventura et al. (2005)

Sánchez-Covisa et al. (1979)

Sarto i Monteys \& Saiz-Ardanaz (2003)

De Prada \& Gil Collado (1959)

Goldarazena et al. (2008)

Sahuquillo Herráiz \& Gil Collado (1982a)

Strobl (1900)

Havelka (1982)

Delécolle \& Ortega (1998)
Anónimo (1992)

De Prada \& Gil Collado (1959)

De Prada \& Gil Collado (1959)

Strobl (1900)

De Prada \& Gil Collado (1959) Goldarazena et al. (2008) 


\section{ESPECIES ORDENADAS} POR SUBGÉNEROS

C. paradisionensis Boorman, 1988

C. pictipennis Staeger, 1839

C. poperinghensis Goethebuer, 1953

C. pseudoheliophilus Callot \& Kremer, 1961

C. pseudopallidus Khalaf, 1961

C. santonicus Callot, Kremer, Rault \& Bach, 1966

C. semimaculatus Clastrier, 1958

C. shaklawensis Khalaf, 1957

C. simulator Edwards, 1939

C. submaritimus Dzhafarov, 1962

C. truncorum Edwards, 1939

C. univitattus Vimmer, 1932

C. vexans Staeger, 1839

C. vidourlensis Callot, Kremer, Molet \& Bach, 1968
AUTORES DE LAS PRIMERAS CITAS PARA ESPAÑA

Pontoculicoides Remm, 1968

C. saevus Kieffer, 1922

C. sejfadinei Dzhafarov, 1958

C. tauricus Gutsevich, 1959

Mellor et al. (1983)

Talavera et al. (2011) González \& Goldarazena (2011)

Silvaticulicoides Glukhova, 1972

C. achrayi Kettle \& Lawson, 1955

C. fascipennis (Staeger, 1839)

C. pallidicornis Kieffer, 1919

C. picturatus Kremer \& Deduit, 1961

González \& Goldarazena (2011)

De Prada \& Gil Collado (1959)

Anónimo (1992)

Delécolle (2002)

Talavera et al. (2011)

Delécolle (2002)

González \& Goldarazena (2011)

Mellor et al. (1983)

Delécolle (2002)

Delécolle (2002)

Sahuquillo Herráiz \& Gil Collado (1982a)

Anónimo (1992)

Sahuquillo Herráiz \& Gil Collado (1982a)

Mellor et al. (1983)

C. subfasciipennis Kieffer, 1919

Capela et al. (1992)

De Prada \& Gil Collado (1959)

Sarto i Monteys \& Saiz-Ardanaz (2003)

Delécolle (2002)

Sahuquillo Herráiz \& Gil Collado (1982a)

Synhelea Kieffer, 1925

C. accraensis Carter, Ingram \& Macfie, 1920

C. coluzzii Callot, Kremer \& Bailly-Choumara, 1970

C. corsicus Kremer, Leberre \& Beaucournu-Saguez, 1971

C. sahariensis Kieffer, 1923

C. yemenensis (Boorman, 1989)

Sahuquillo Herráiz \& Gil Collado (1982a)

Talavera et al. (2011)

Gil Collado \& Sahuquillo Herráiz (1983)

Sahuquillo Herráiz \& Gil Collado (1982b)

Talavera et al. (2011)

Wirthomyia Vargas, 1973

C. minutissimus (Zetterstedt, 1855)

C. segnis Campbell \& Clinton, 1959

Sahuquillo Herráiz \& Gil Collado (1982a)

Gil Collado \& Sahuquillo Herráiz (1983)

\section{Indefinido}

C. bahrainensis Boorman, 1989

C. indistinctus Khalaf, 1961

C. marcleti Callot, Kremer \& Basset, 1968

C. odiatus Austen, 1921

C. paolae Boorman, 1996

Ortega (1993)

Talavera et al. (2011)

Ortega (1993)

Mellor et al. (1983)

Estrada et al. (2011)

Nomen dubium

C. analis Santos Abreu, 1918

Borkent (1997)

los casos), tiempo más que suficiente para que otros autores expongan novedades que no hayan podido ser tenidas en cuenta en un principio. Por tanto y demás, es obvio que estas aportaciones pueden considerarse válidas al tener en cuenta el contexto temporal de cada una de ellas, si bien con la literatura en mano las autorías deben ser concedidas al trabajo publicado con más anterioridad.
En conclusión y a día de hoy, hay descritas en España 81 especies de Culicoides, repartidas en 9 subgéneros, un grupo misceláneo (subgénero indefinido) y un nomen dubium (Tabla 1). A pesar de ello y debido a la existencia de especies crípticas, se considera preciso desarrollar técnicas moleculares específicas que permitan separar con claridad las especies para poder esclarecer las dudas sobre 
diferentes sinonimias aparentes, pues existen variaciones intraespecíficas importantes que añaden, más si cabe, gran complejidad al grupo.

\section{Agradecimientos}

Los autores desean expresar su más sincero agradecimiento a los colegas Mikel González González (NEIKER-Instituto Vasco de Investigación y Desarrollo Agrario) y David Borrás (Universitat de les Illes Balears) por proporcionarnos valioso material bibliográfico sobre el estudio de estos dípteros.

\section{Referencias}

Anónimo, 1992. Study on the geographical distribution and seasonal prevalence in Spain during 1990-1991 of different species of the genus Culicoides (Family Ceratopogonidae). Documento científico-técnico restringido, documento VI/650/92: Ministerio de Agricultura, Pesca y Alimentación. Madrid. 57 pp.

Arias Encobet, J., 1912. Datos para el conocimiento de la distribución de los Dípteros de España. Memorias de la Real Sociedad Española de Historia Natural, 7: 61-246.

Blackwell, A., Wadhams, L. J. \& Mordue, W., 1997. Electrophysiological and behavioural studies of the biting midge, Culicoides impunctatus Goetghebuer (Diptera, Ceratopogonidae): interactions between some plant-derived repellent compounds and a hostodour attractant, 1-octen-3-ol. Physiological Research, 82: 299-306.

Boorman, J., 1989. Culicoides (Diptera: Ceratopogonidae) of the Arabian Peninsula with notes on their medical and veterinary importance. Fauna of Saudi Arabia, 10: 160-224.

Borkent, A., 1997. The Ceratopogonidae (Diptera) described by Santos Abreu from the Canary Islands. Deutsche Entomologische Zeitschrift, 44:3-18.

Callot, J., Kremer, M., Geiss, J-L., Delécolle, J-C., \& Hommel, M., 1975. Notes d'Entomologie. Localisations nouvelles de Culicoides (Dipteres, Ceratopogonides). Annales de Parasitologie Humaine et Comparée, 50(2): 245-246.

Capela, R., Pena, I. \& Kremer, M., 1992. Contribucao para o conhecimento dos Culicoides (Diperta, Ceratopogonidae) existentes em Portugal. Actas do V Congresso Iberico de Entomologia, 381-386 pp.

Czerny, L. \& Strobl, P. G., 1909. Spanische Dipteren. III. Verhandllungen der kaiserlich-kongiglichen zoologisch-botanischen Gesellschaft in Wien, 59(6): 121310.

De Prada, J. \& Gil Collado, J., 1959. Culicoides in Badajoz. La Medicina Tropical, 34: 417-424.
Delécolle, J-C. \& Ortega, M. D., 1998. Description d'une espéce nouvelle du genre Culicoides originaire d'Espagne, apparentée á C. fagineus Edwards, 1939 (Diptera, Ceratopogonidae). Nouvelle Revue Entomology, 15: 283-290.

Delécolle, J-C., 2002. Ceratopogonidae. Catálogo de los Diptera de España, Portugal y Andorra (Insecta). Monografias de la Sociedad de Entomologia Aragonesa, Zaragoza, 8, 23-26.

Delécolle, J-C., Blasco-Zumeta, J. \& Rieb, J. P., 1997. Nouvelle contribution à l'étude des Cératopogonidés d'Espagne. Description de Homohelea iberica n.sp. et redescription de Palpomyia miki Goetghebuer, 1934 et de Culicoides brevifrontis Smatov \& Isimbekov, 1971 (Diptera, Nematocera). Nouvelle Revue Entomology, 14(4): 337-351.

Estrada, R., Carmona, V. J., Alarcón-Elbal, P. M., Miranda, M. A., Borrás, D., Roche, M. L., Tamarit, A., Navarro, J. \& Lucientes, J., 2011. Primera cita de Culicoides paolae Boorman, 1996 (Diptera, Ceratopogonidae) para la Península Ibérica. Boletín de la Sociedad Entomológica Aragonesa (S.E.A.), 49: 217-221.

Gil Collado, J. \& Sahuquillo Herráiz, C., 1983. Aportaciones al catálogo de Culicoides (Diptera, Ceratopogonidae) de España peninsular. Revista Ibérica de Parasitología, 43: 109-110.

Gil-Collado, J. \& Sahuquillo Herráiz, C., 1985. Claves de identificación de Ceratopogonidae de España peninsular. II. Subfamilia Ceratopogoninae (Dip., Nematocera). Graellsia, 41: 43-63.

Goldarazena, A., Romón, P., Aduriz, G., Balenghien, T., Baldet, T. \& Delécolle J-C., 2008. First record of Culicoides imicola, the main vector of bluetongue virus in Europe, in the Basque Country (Northern Spain). Veterinary Record, 162: 820-821.

González, M. \& Goldarazena, A., 2011. El género Culicoides en el País Vasco. Guía Práctica para su identificación y control. Ed. Servicio Central de Publicaciones del Gobierno Vasco. 247 pp.

Gorla, D. E., 2002. Variables ambientales registradas por sensores remotos como indicadores de la distribución geográfica de Triatoma infestans (Heteroptera: Reduviidae). Ecología austral, 12(2): 117-127.

Havelka, P., 1982. Neue Ceratopogonidenfunde von der Iberischen Halbinsel. Eos, 58: 47-134.

Meiswinkel, R., Baldet, T., De Deken, R., Takken, W., Delécolle, J-C. \& Mellor, P. S., 2008. The 2006 outbreak of bluetongue in northern Europe-The entomological perspective. Preventive Veterinary Medical, 87 (1-2): 55-63.

Meiswinkel, R., Labuschagne, K. \& Goffredo, M., 2004. Christopher Columbus and Culicoides: was $C$. jamaicensis Edwards, 1922 introduced into the Mediterranean 500 years ago and later re-named $C$. paolae Boorman 1996? Veterinaria Italiana, 40(3): 340-344. 
Mellor, P. S., Boorman, J. P. T., Wilkinson, P. J. \& Martinez-Gómez, F., 1983. Potential vectors of bluetongue and African horse sickness viruses in Spain. Veterinary Record, 112: 229-230.

Ministerio de Agricultura, Alimentación y Medio Ambiente (MAGRAMA), 2012. Informe sobre la situación epidemiológica del virus de Schmallenberg. Marzo 2012. 12 pp.

Organización Mundial de Sanidad Animal (OIE). (2010). Enfermedades animales. Lengua Azul. Ficha técnica. Disponible en: http://www.oie.int/. Última actualización 12-Junio del 2010.

Ortega, M. D., 1993. Distribution and frequency of the Culicoides spp. (Diptera: Ceratopogonidae) in Andalucía, Spain. $37^{\text {th }}$ Annual Livestock Insect Workers Conference. Santa Fe, New Mexico, USA, 3 pp.

Ortega, M. D., Mellor, P. S., Rawlings, O. \& Pro, M. J., 1999. The seasonal and geographical distribution of Culicoides imicola, C. pulicaris group and C. obsoletus group biting midges in central and southern Spain. Archives of Virology, 14: 85-91.

Rawlings, P., 1996. A key, based on wing patterns of biting midges (Genus Culicoides Latreille-Diptera: Ceratopogonidae) in the Iberian peninsula, for use in epidemiological studies. Graellsia, 52: 52-71.

Remm, H., 1981. New synonyms and new names of the Palaearctic Ceratopogonidae (Diptera). Eesti NSV Teaduste Akadeemia Toimetised, 30: 27-32.

Romón, P., Higuera, M., Delécolle, J-C., Baldet, T., Aduriz, G. \& Goldarazena, A., 2012. Phenology and attraction of potential Culicoides vectors of bluetongue virus in Basque Country (northern Spain). Veterinary Parasitology, 186: 415-424.

Sahuquillo Herráiz, C., 1981. Ceratopogonidae (Diptera, Nematocera) de España peninsular. $\mathrm{PhD}$ thesis, Universidad Complutense, Madrid, España.

Sahuquillo Herráiz, C. \& Gil Collado, J., 1982a. Ceratopogonidae (Diptera nematocera) de Navarra. Revista de Sanidad e Higiene Pública, 56: 743-752.

Sahuquillo Herráiz, C. \& Gil Collado, J., 1982b. Culicoides sahariensis Kieffer, 1923 (Diptera ceratopogonidae), nueva especie para Europa y España. Revista Ibérica de Parasitología, 42: 241-242.

Sánchez-Covisa, A., Rodríguez Rodríguez, J. A., Pascual Sánchez-Gijón, E., 1979. Notas sobre Heleidae (Ceratopogonidae) arborícolas de los alrededores de Madrid (Diptera, Nematocera). Revista Ibérica de Parasitología, 39: 515-257.

Santos Abreu, E., 1918. Ensayo de una Monografía de los Tendipedidos de las Islas Canarias. Memorias de la Real Academia de Ciencias y Artes de Barcelona, 14(2): 159-326.

Sarto i Monteys, V., Delécolle, J-C., Moreno-Vidal, J. O. \& Pinna, M., 2009. New records of biting midges of the genus Culicoides Latreille (Diptera: Ceratopogonidae) for Spain and Catalonia Autonomous Community (NE Spain), with notes on their biology and veterinary importance. Proceedings of the Entomological Society of Washington, 111(3): 714-733.

Sarto i Monteys, V. \& Saiz-Ardanaz, M., 2003. Culicoides midges in Catalonia (Spain), with special reference to likely bluetongue virus vectors. Medical and Veterinary Entomology, 17: 288-293.

Strobl, P. G., 1900. Spanische Dipteren, XI. Theil, Wiener Entomologische Zeitung, 19: 169-174.

Strobl, P. G., 1906. Spanische Dipteren. II: Beitrag. Memorias de la Real Sociedad Española de Historia natural, Tomo III, Memoria $5^{a}$ (1905) 271-422.

Takken, W. \& Knols, B. G. J., 2010. Olfaction in vectorhost interactions. Ecology and Control of VectorBorne Diseases, 2(10): 217-246.

Talavera, S., Muñoz-Muñoz, F. \& Pagès, N., 2011. New insights on diversity, morphology and distribution of Culicoides Latreille 1809 (Diptera: Ceratopogonidae) from Northeast Spain. Annales de la Société Entomologique de France, 47(1-2): 214-231.

Ventura, D., Pagès, N. \& Sarto, V., 2005. Citas nuevas e interesantes de Culicoides Latreille, 1809 para Cataluña y la Península Ibérica (Diptera, Nematocera, Ceratopogonidae). Boletín de la Asociación Española de Entomología, 29: 75-86.

\section{RESUMEN}

El número de estudios acerca de los artrópodos del género Culicoides Latreille (Diptera, Ceratopogonidae) en España ha experimentado un elevado incremento en las últimas décadas. Principalmente ello es debido al papel que estos dipteros juegan como vectores de arbovirus causantes de enfermedades en los animales, aunque también en humanos. Este trabajo acomete una actualización del catálogo de las especies que conforman este género en nuestro país, mediante una revisión crítica de la literatura existente, clarificando aspectos cronológicos sobre estas publicaciones llevadas a cabo durante más de un siglo de investigación. Esta actualización muestra un total de 81 especies de Culicoides para España, entre las que se encuentran algunas a tener en cuenta por estar directamente relacionadas con la trasmisión de enfermedades como la Lengua Azul o la Peste Equina Africana.

Palabras clave: catálogo, Culicoides, Diptera, arbovirus, actualización, España. 


\section{ABSTRACT}

An updated catalogue of the Culicoides Latreille, 1809 (Diptera, Ceratopogonidae) of Spain.

The number of studies on arthropods of genus Culicoides Latreille (Diptera, Ceratopogonidae) has increased considerably in Spain in recent decades. This is due to the role these insects play as vectors of arboviruses that cause disease in animals, but also in humans. This work undertakes an updated catalogue of the species of this genus in our country, through a critical review of the literature, clarifying chronological aspects of these publications carried out for over a century of research. This update shows a total of 81 species of Culicoides in Spain, among which are some to be considered as directly related to the transmission of diseases such as bluetongue and African horse sickness.

Keywords: catalogue, Culicoides, Diptera, arbovirus, update, Spain.
Recibido / Received, 21-03-2012 Aceptado / Accepted, 5-06-2012
Publicado en línea / Published online, 1-08-2012 Publicado en línea / Published online, 1-08-2012
Publicado impreso / Published in print, 31-12-2012 